\title{
CAS in Manned Space Flights and Lunar Exploration
}

$\mathrm{T}$ The Chinese Academy of Sciences (CAS) is among the initiators of the Manned Space Flight

Program (MSFP) of China as well as the China Lunar Exploration Program (CLEP), and has directly contributed to these grand programs. The Academy proposed the scientific goals of these programs: as a coorganizer and important contributor, it has overseen the implementation of the Programs to secure the fulfillment of the preset goals, providing strong S\&.T support. During the whole process, institutions under its administration have undertaken a lot of important tasks from these programs. Their participation in these historic missions has led to breakthroughs in many fields.

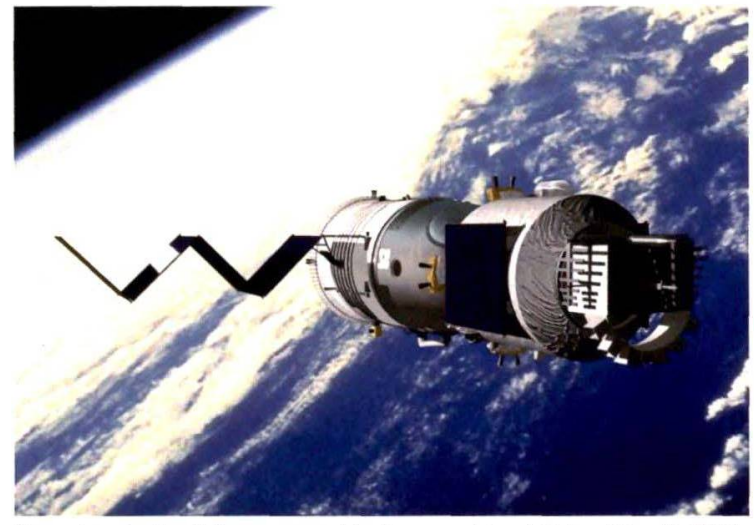

Shenzhou-1, the first unmanned test spaceship of China (Credit: CSU)

\section{Manned Space Flight Program of China}

In the implementation of manned space flights, the Academy takes charge of the R\&D and operation of the space application systems. Working to this end, the General Establishment of Space Science and Application
(GESSA) was set up under the CAS Center for Space Science and Applied Research (CSSAR, now the National Space Science Center, CAS) to oversee the organization. administration and coordination of related tasks. GESSI

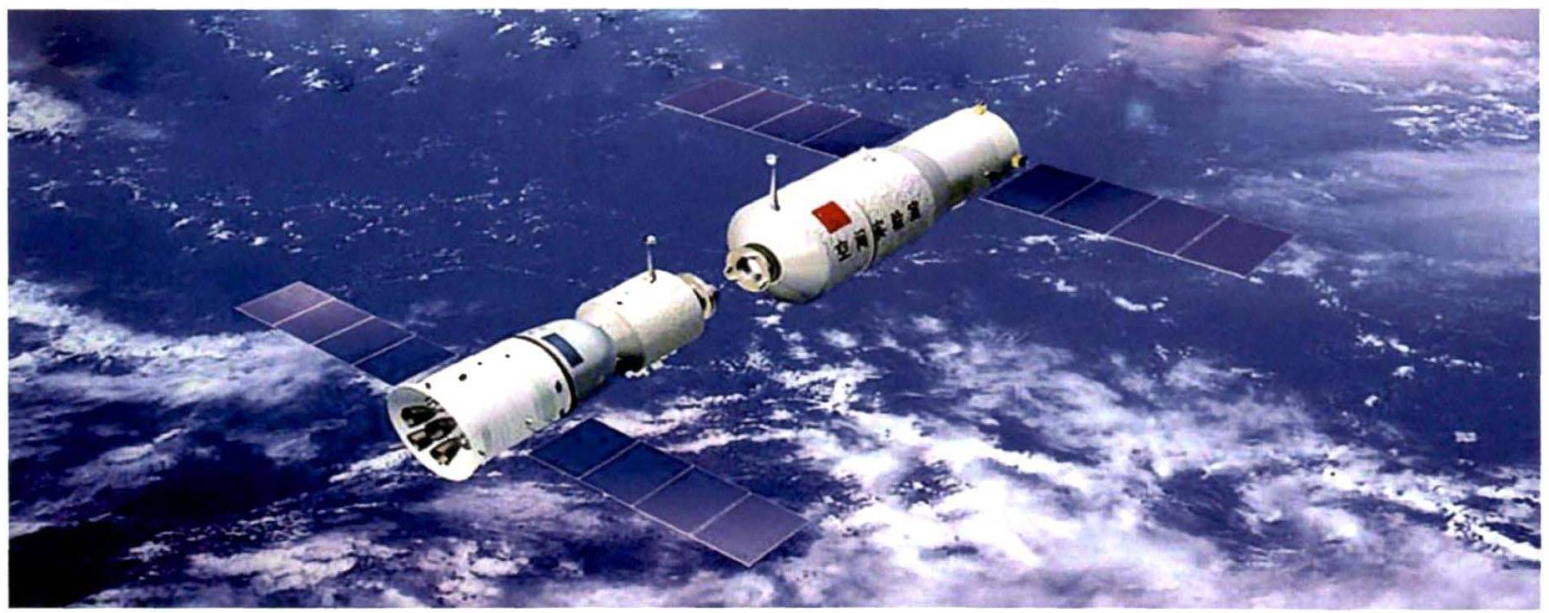

The Tiangong- 1 and the Shenzhou- 8 successfully dock on each other in orbit. 


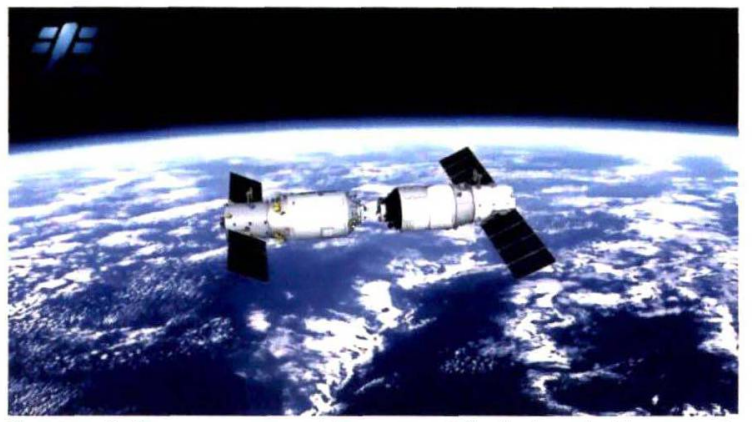

Tianzhou-1, the cargo spaceship, successfully docks onto Tiangong-2, the space lab of China. (Credit: CMS/CSU)



Application system aboard the Tiangong-2 space lab (Credit: CSU)

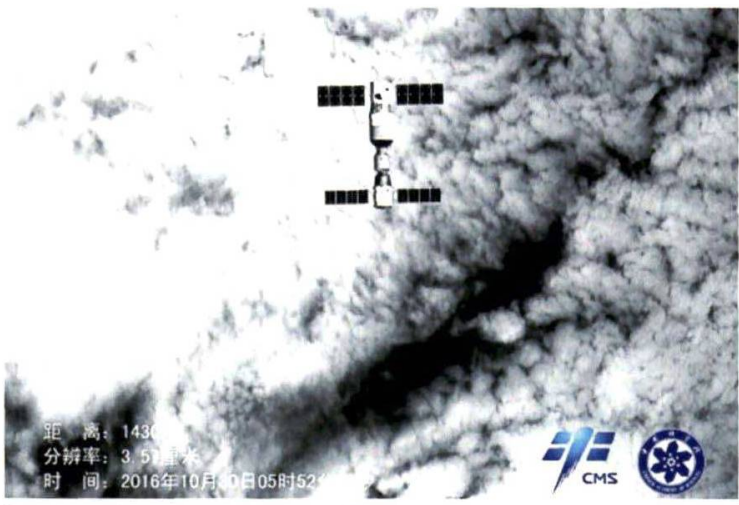

The in-orbit docking between Chinese spaceship Shenzhou-11 and the space lab Tiangong-2, as captured by a camera aboard an accompanying satellite sent from the spaceship. (Credit: CMS/CAS)

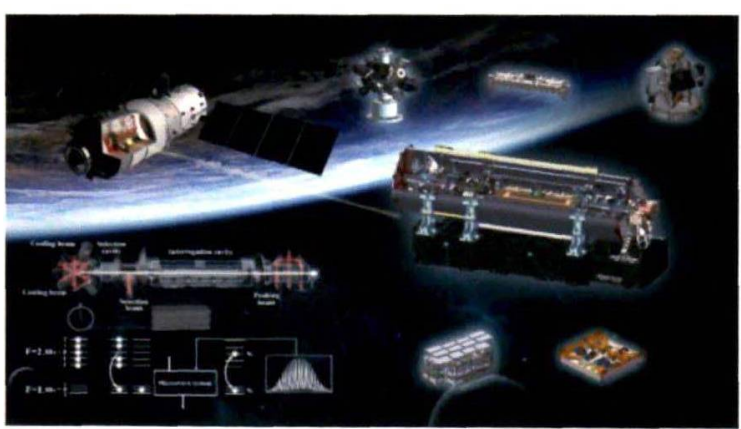

The functional structure and working principle of the space cold atomic clock (Credit: CSU) was later upgraded and renamed the Technology and Engineering Center for Space Utilization (CSU). CAS. So far, involved institutions of CAS have accomplished over 70 tasks of space science and application. and over 560 tasks for the RdD of payloads, including those involved in the missions of Shenzhou serial spaceships. the Tiangong-1 and Tiangong-2 space lab modules. and the cargo spacecraft Tianzhou-1.

Resulting from the continued innovation and development. the Academy has advanced technologies for visible light, infrared and hyperspectral imaging, and technologies for microwave remote sensing, promoting the advancement of Earth observation in China. She has spearheaded space experimental research in a series of disciplines, including life sciences, microgravity fluid science, material science, fundamental physics. and astronomy.

The Academy has thus marked milestones in China's manned aerospace cause. In November 1999, Shenzhou-1, the first unmanned test spaceship of China. was thrown into space from the Jiuquan Satellite Launch Center in northwestern China. Its 21 hour flight verified the data managing module of the space application system and laid a foundation for the upcoming missions of MSFP. Later in 2003. the first manned flight of the Program performed by Shenzhou-5.5 caught the attention of the whole world with its success.

In 2008, in the third manned space flight of China. Shenzhou-? successfully carried three astronauts altogether into space. This flight not only saw an astronaut safely floating out of the cabin to conduct a spacewalk, but also released an accompanying satellite in orbit. This marked the first time of China to release a satellite from a spacecraft flying in outer space. On November $1^{\text {st }}$ of 2011. Shenzhou-8 was sent into space from the Jiuquan Satellite Launch Center. During its long journev, the spaceship successfully conducted two automatic in-orbit docking operations with the space lab Tiangong-1, respectively on November $3^{\text {rd }}$ and $14^{\text {th }}$. This feat made China the third in the world to master the technology needed for in-orbit docking.

In 2016. CAS scientists accomplished 1t scientific experiments aboard Tiangong-2, a space lab module of China. Among them. the space cold atomic clock succeeded in improving the timing accuracy by 12 orders of magnitude, reaching an important milestone in the development of space quantum science. 


\section{Lunar Exploration Program}

CAS also plays a role of objective-setter for CLEP. The Academy proposed the scientific goals of the program and layout, and sees to its implementation with support from its $100^{+}$institutes spreading across the country. Represented by the National Astronomical Observatories, CAS (NAOC), involved CAS institutions have taken charge of R\&D of the ground application system, the onboard payloads, the precise orbit determination with its Very Long Baseline Interferometry (VLBI) network, the engineering support system as well as related key modules, and the analysis of the scientific data.

Since 2004, CAS has successfully accomplished the engineering R\&D and scientific detection involved in the missions of Chang'e-1, 2, 3 and 4 , breaking through in many fields, including the data transfer to and from the Moon. accurate measurement and determination of the orbit of the spacecraft using VLBI network, the R\&D of payloads, and processing and analysis of the data produced from the missions.

Since the first mission of CLEP, CAS as an important contributor has notched a lot of achievements of international importance. For example, based on the data obtained by Chang'e-2, scientists for the first time revealed the distribution of microwave radiation brightness temperatures on the lunar surface; based on the data from Chang'e-3, scientists discovered a new type of rock near the landing site, and reconstructed possible geological evolution of this area.

CLEP caught the eye of the whole world in January $9^{\text {th }}, 2019$, when Yutu-2, the rover of Chang'e-t mission successfully performed a soft-landing on the far side of the Moon - the first time ever for man-made spacecraft to do so. Queqiao - the relay satellite that bridged the gap in data transmission from the lunar far side to the Earth, also

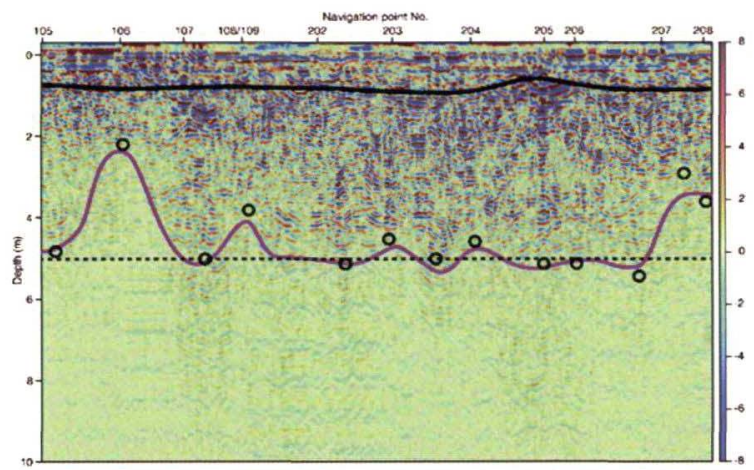

A chart showing the characteristics of the superficial structure of the landing site of Chang'e-3 (Credit: OLDE/CAS)

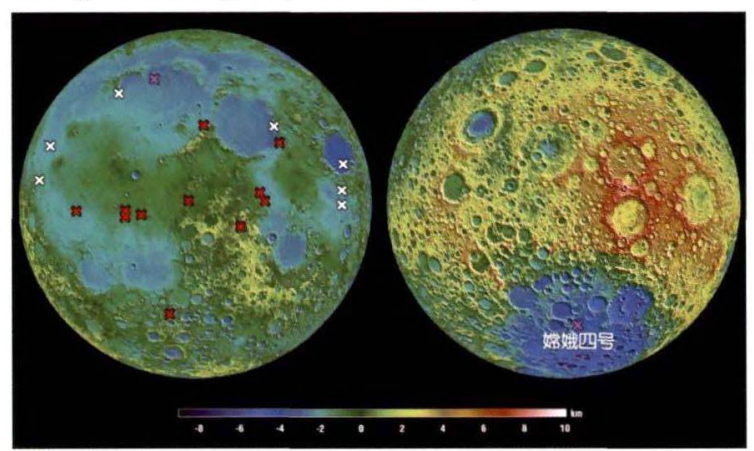

Before Chang'e-4, all lunar rovers landed on the near side of the Moon (left). On the right shown is the landing site of Chang'e-4. (Credit: NASAVGSC/MIT

represents an innovative breakthrough in data transfer.

The overall development layout of China Lunar Exploration Program (CLEP) is divided into three major stages, with the first focusing on robotic exploration, the second on manned landing, and the third on human residence. The current stage of CLEP (from 2004 till 2020 ) is named Chang'e Program after a legendary fairy dwelling on the Moon in Chinese mythology. For more about CLEP, including its middle- and long-term layout, please refer to Page 72, Vol. 33, No.2 of BCAS.

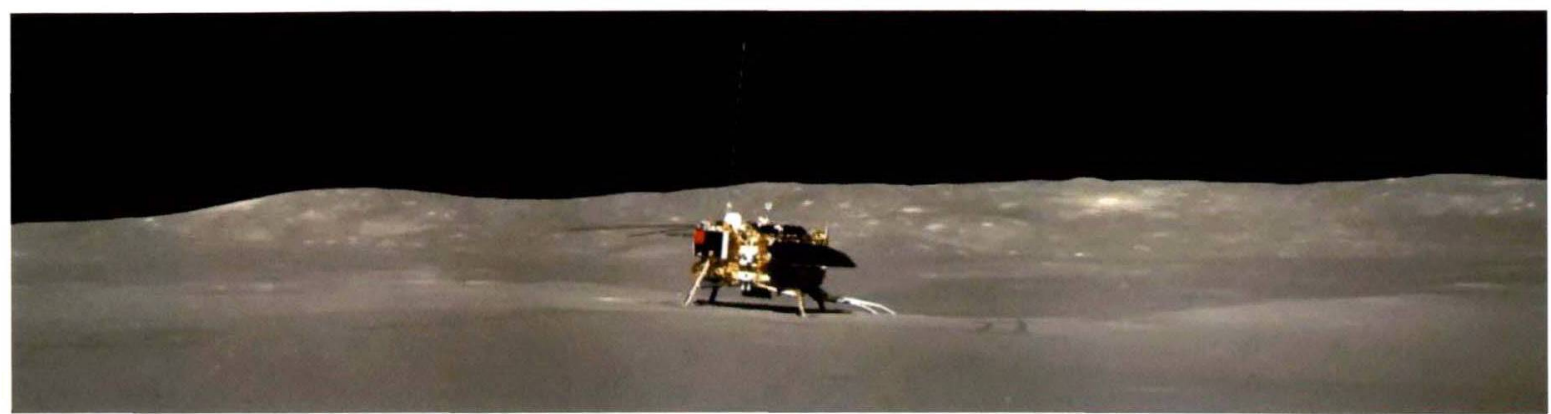

On January 9, 2019, Yutu-2, the lunar rover of Chang'e-4 mission, landed softly at the Von Kármán crater on the far side of the Moon. (Credit: CLEP/GRAS) 